\title{
Emerging clinical significance of claudin-7 in colorectal cancer: a review
}

This article was published in the following Dove Press journal:

Cancer Management and Research

\section{Kun Wang \\ Chang Xu \\ Wenjing Li \\ Lei Ding}

Department of Oncology, Beijing Shijitan Hospital, Capital Medical University, Beijing, 100038, People's Republic of China
Correspondence: Lei Ding Department of Oncology, Beijing Shijitan Hospital, Capital Medical University, Tieyilu 10 Yangfangdian, Haidian, Beijing, 100038, People's Republic of China Tel +86 I 35527782 I।

Email dingleil005@।26.com

\begin{abstract}
Tight junctions (TJs) play an important role in maintaining cell polarity and regulating cell permeability. In recent years, many studies have shown that $\mathrm{TJ}$ proteins, especially claudin-7, are closely related to inflammation and the development of various malignant tumors. Claudin-7 plays a significant role in maintaining the physiological functions and pathological conditions of the TJ barrier. The dysregulation of claudin-7 plays a tumor suppressor role or conversely has carcinogenic effects in different target tissues or cells, but the exact underlying mechanism is still unclear. In this review, we will summarize the expression pattern of claudin-7 in tumors, focusing on the expression and regulation of claudin-7 in colorectal cancer and discussing the correlation between claudin-7 and invasion, metastasis and epithelial-mesenchymal transition (EMT) in colorectal cancer. The construction of $\mathrm{Cldn}^{-/-}$mice and conventional claudin-7 knockout mouse models has helped determine the mechanisms by which claudin-7 promotes tumorigenesis. Elucidation of the expression and subcellular localization of claudin-7 under pathological conditions will help develop claudin-7 as a useful biomarker for detecting and diagnosing cancer, and thus may help combat the occurrence, development, and invasion of cancers.
\end{abstract}

Keywords: tight junctions, claudin-7, tumors, colorectal cancer, invasion, metastasis

\section{Introduction}

The incidence of tumors is increasing worldwide. Colorectal cancer (CRC) is a common malignant gastrointestinal cancer. The morbidity and mortality are ranked third and fourth among malignant tumors, respectively. ${ }^{1,2} \mathrm{CRC}$ is a serious threat to human health. With the improvement of economic conditions and human living standards, the incidence of CRC has increased, and its morbidity and mortality are gradually rising in China. The current incidence and mortality rates are ranked third and fifth in malignancies, respectively. ${ }^{1,3}$ Approximately, 700,000 patients die each year from $\mathrm{CRC}$, which is related to poor prognosis, high recurrence rates, invasion, and metastasis of CRC. ${ }^{4}$

Increasing evidence has indicated that the abnormal structure and functional disruption of cell-cell tight junctions (TJs) is one of the important mechanisms by which malignant tumor cells can easily detach from primary cancer tissues and cause distant invasion and metastasis. ${ }^{5}$ Claudin proteins are the most crucial components of TJs, and their core functions include regulating the intercellular ion and small molecule flux, maintaining the epithelial cell polarity, ${ }^{6,7}$ and participating in cell proliferation and differentiation, gene transcription, and signal transduction processes.$^{8-10}$ The expression profiles of claudins change significantly in many epithelial-derived tumors, and the 
expression correlates with tumor prognosis and can serve as a biomarker of prognosis and potential therapeutic targets. ${ }^{5}$

In recent years, many studies of the expression and regulatory mechanism of claudin-7 in tumors have been reported. Claudin-7 is a member of claudin family and is an important molecule that constitutes cell-cell TJs. This protein plays important roles in maintaining cell polarity and barrier functions. The abnormal expression of claudin- 7 results in the destruction of TJs, loss of the contact inhibition of cells, and abnormal proliferation and migration, and is closely related to the occurrence and development of various malignant tumors. ${ }^{11}$ This review will summarize the current knowledge regarding claudin-7 dysregulation in cancer and highlight the progress in claudin-7 research in CRC.

\section{Introduction to tight junctions and claudin proteins physiology}

TJs are apical intercellular junctions between epithelial cells and vascular endothelial cells. The stability of the TJs requires the coordinated activity of several different proteins, including occludin, claudin, junctional adhesion molecule, and TJ-associated proteins (ZO-1, ZO-2, and ZO-3). ${ }^{8}$ The growth of epithelial cells is mediated by cell-cell communication, such as cell adhesion and TJs. The apical membrane forms a semipermeable barrier that allows only ions and solutes selectively through epithelial cells. ${ }^{12}$ The primary functions are regulating the cell permeability through its barrier function and maintaining the cell polarity by limiting the diffusion of lipids and proteins through its fence function. ${ }^{13-15}$ Nearly $90 \%$ of malignant tumors originate from the epithelium. ${ }^{8}$ The destruction of TJ integrity between epithelial cells is an important step in tumor progression. This alteration weakens cell-cell adhesion and promotes the spread of growth factors and cytokines, leading to the loss of tumor cell polarity, uncontrolled growth, and eventually tumor invasion and metastasis. $5,8,15,16$

Claudins are the main components of TJs and regulate barrier properties by controlling the charge and molecular size selectivity. Claudins are a multigene family with a relative molecular mass of $22-27 \mathrm{kDa} .{ }^{16}$ There are currently 27 identified claudin members, in humans and mice. The expression pattern of claudins shows high tissue and organ specificity. ${ }^{17,18}$ Claudin family members have similar chemical structures and are composed of four hydrophobic transmembrane domains and two extracellular loops, with the amino and carboxyl terminals located within the cells. ${ }^{19,20}$ The first extracellular loop contains two highly conserved cysteine residues that increase protein stability through disulfide bond formation and determine the transepithelial electrical resistance of TJs and selectivity of paracellular pathways. ${ }^{21}$ The other extracellular loop is short and forms a dimer between the conserved aromatic residues and the claudin proteins. ${ }^{22}$ The carboxyl terminus constitutes the PDZ domain (PSD-95/ Dlg/ZO-1) and has potential phosphorylation sites, such as sites for serine, threonine, and tyrosine phosphorylation, and palmitoylation, which can affect the localization and function of claudin proteins in TJ complexes. ${ }^{23,24}$

Claudin family members form pores to control the extracellular fluid and ions in epithelial cells; in addition to their role in barrier regulation, they also maintain epithelial cell homeostasis. ${ }^{25}$ The dysregulation of claudin proteins and disruption of epithelial barrier function have been observed in intestinal inflammation and tumors. Downregulation or absence of claudin proteins was identified as an important mechanism underlying loss of cell adhesion and metastasis, and resulted in structural destruction and impaired function of epithelial and endothelial cells. ${ }^{5,8}$ The expression of claudin-1, -2 , and -18 in intestinal mucosal epithelium was increased in intestinal inflammatory diseases, with claudin-3, $-4,-5,-7,-8$, and -12 being downregulated. ${ }^{25}$ Claudin- 1 is widely known for its barrier function in intestinal epithelial cells. Overexpression of claudin-1 in mouse intestinal epithelial cells resulted in upregulation of matrix metalloproteinase 9 (MMP9) and extracellular signal-regulated kinase (ERK). Claudin-1 plays a key role in colonic epithelial cell homeostasis by regulating the Notch signaling pathway. ${ }^{26}$ The expression of claudin-1 was significantly reduced in inflammatory bowel disease (IBD) patients and was associated with the course of the disease. ${ }^{27}$ Claudin-2 and -15 regulate the paracellular transport of $\mathrm{Na}^{+}, \mathrm{Ca}^{2+}$, and water in the intestine. Deletion of claudin-2 and -15 in mouse intestinal epithelial cells resulted in intestinal destruction because the transepithelial resistance and paracellular $\mathrm{Na}^{+}$ permeability were decreased. Specific overexpression of claudin-2 resulted in increased growth of colonic crypts and intestinal dilatation. ${ }^{28,29}$

In this study, the specific contribution of claudin-7 to maintenance of normal epithelial cell homeostasis and tumorigenesis is being explored. Claudin-7 is necessary to maintain the mucosal epithelial integrity and the $\mathrm{NaCl}$ balance in distal nephrons. Overexpression of claudin-7 reduces the paracellular $\mathrm{Cl}^{-}$permeability and increases the paracellular $\mathrm{Na}^{+}$conductance in renal LLC-PK1 cells, whereas claudin-7 knockdown increases $\mathrm{Na}^{+}$permeability in MDCK cells. ${ }^{30} \mathrm{Cl}^{-}$permeability was increased in the claudin-7 knockout mouse model, leading to severe loss of $\mathrm{Na}^{+}, \mathrm{Cl}^{-}$, and $\mathrm{K}^{+}$as well as chronic dehydration 
and growth retardation. The paracellular flux (pFlux) of small organic solutes was enhanced by claudin-7 deficiency, but not for the larger organic solutes, resulting in increased infiltration of N-formyl-L-methionyl-L-leucyl-Lphenylalanine (438 Da), a major bacterial product, and aggravated intestinal inflammation. ${ }^{31-33}$ These studies suggest that claudin-7 is required for the maintenance of intestinal epithelial barrier function by modifying intestinal permeability and regulating isohydria in the kidney. Immunofluorescence analyses showed decreased staining and signaling of claudin-7 in ulcerative colitis (UC) patients. Downregulation of claudin-7 may lead to epithelial damage and $\mathrm{TJ}$ structural change, which disrupts the paracellular pathway selectivity and promotes UC progression. ${ }^{34}$ Claudin-7 expression is also associated with a variety of malignancies, but the exact mechanism is still unclear.

\section{The localization and function of claudin-7}

Claudin-7 is one of the most important members of the claudin family; it consists of 211 amino acid residues and is widely distributed in the head and neck, gastrointestinal tract, lung, breast, kidney, bladder, and skin. ${ }^{35}$ Claudin-7 is not only localized at apical intestinal epithelial cells but also has a strong basolateral and basement membrane distribution in the intestines. In contrast to other TJ proteins, which are usually located only on the top of intestinal epithelial cells, claudin-7 has unique cell distribution characteristics (Figure 1). ${ }^{31,36}$ At the same time, claudin-7 mRNA and protein are strongly expressed in the intestine. ${ }^{31}$ In the colon, claudin-7 expression gradually increases with the differentiation of epithelial cells into the luminal surface, which results in an expression gradient from the basal surface to the surface of the intestinal lumen. ${ }^{37}$

The barrier function of claudin-7 restricts the supply of nutrients and growth factors of cancer cells and plays a tumor suppressor role. ${ }^{38}$ Claudin-7 deletion leads to TJ loss, barrier function disruption, and destruction of the electrochemical gradient, which can result in the transfer of toxins and pathogens to the colon. ${ }^{33}$ The basolateral membrane expression of claudin-7 in intestinal epithelial cells maintains extracellular matrix interactions, homeostasis, and differentiation through signal transduction. ${ }^{31,39}$ Claudin-7 colocalizes with integrin $\alpha 2$ in normal intestinal epithelium, and deletion of claudin-7 altered integrin $\alpha 2$ expression and localization and decreased

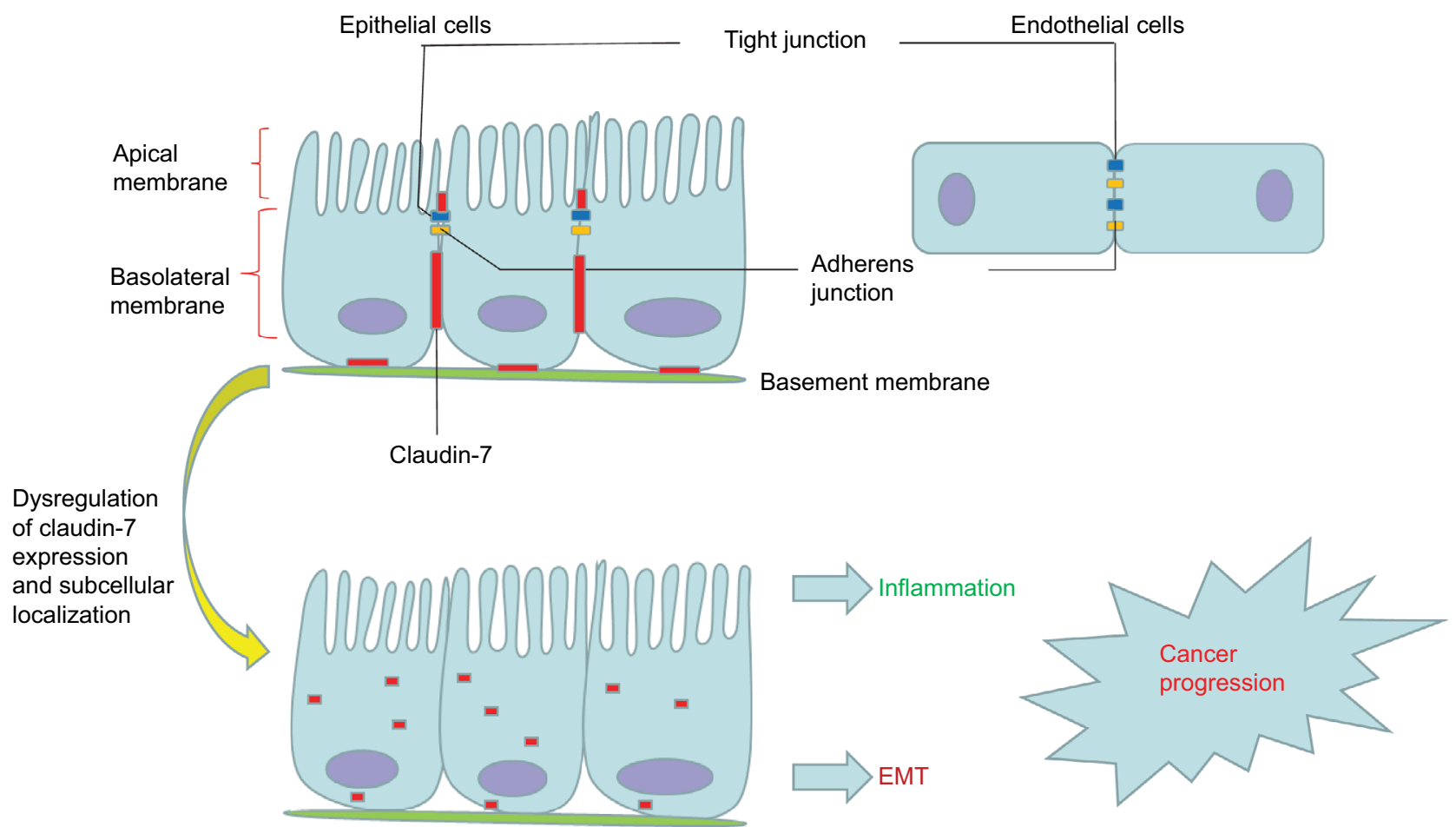

Figure I The junctional complex in epithelial and endothelial cells.

Notes: Tight junctions (blue) and adherens junctions (yellow) are apically located in polarized epithelial cells and endothelial cells. Claudin-7 (red) is not only localized at apical intestinal epithelial cells but also has a strong basolateral and basement membrane distribution in the intestines. Dysregulation of claudin-7 expression and subcellular localization can promote inflammation, EMT, and cancer progression. Abbreviation: EMT, epithelial-mesenchymal transition. 
the claudin-7/integrin $\alpha 2 /$ claudin- 1 protein complex formation. Polymerase chain reaction (PCR) revealed a significant upregulation of MMPs, which degrade the extracellular matrix, destroying intestinal epithelial tissue and causing intestinal inflammation similar to IBD symptoms. ${ }^{31}$ Claudin-7 also colocalizes with integrin $\beta 1$ to form a stable complex in lung cancer. With the inhibition of claudin-7, cell growth was significantly accelerated, the expression of integrin $\beta 1$ and phosphorylated focal adhesion kinase (FAK) was significantly decreased, and extracellular matrix adhesion was weakened, which led to a weakened adhesion capacity of cancer cells, thereby accelerating invasion and metastasis. ${ }^{40}$

\section{Specific function of claudin-7 in the intestine revealed by claudin-7 gene- deficient mouse models}

For analysis of the molecular biological effects of the claudin-7 in intestinal tumorigenesis and development, several claudin-7 knockout mouse models were successfully constructed. Ding et $\mathrm{al}^{31}$ generated systemic claudin-7 gene knockout in mice and found that the general morphology of $\mathrm{Cldn} 7^{-/-}$mice was significantly impaired compared with that of the control group; the integrity of the intestinal epithelial tissue was severely disrupted; and an inflammatory response similar to that in IBD, including mucosal ulceration, epithelial cell sloughing, and inflammatory reactions, was observed, leading to extensive local hyperplasia. Real-time PCR and Western blot (WB) results showed that MMPs and inflammatory cytokines were upregulated, leading to degradation of extracellular matrix components and stimulation of immune responses. Deletion of claudin- 7 reduces the abnormal expression and position of integrin $\alpha 2$, which destroys the claudin-7/integrin $\alpha 2 /$ claudin-1 complex formation in the intestinal epithelium. Electron microscopy analysis showed obvious intercellular gaps between the intestinal cells and cell matrix, suggesting that claudin-7 plays an important role in maintaining the function and homeostasis of intestinal epithelial cells and regulating cell-matrix interactions through non-TJ functions.

Tanaka et $\mathrm{al}^{33}$ constructed a conditional intestinal claudin-7 knockout mouse model using the Cre-LoxP system. Hematoxylin-eosin (H\&E) staining showed that the apoptotic rate of epithelial cells was significantly elevated, neutrophil infiltration increased, and the mRNA levels of proinflammatory cytokines were significantly increased in the ileum and colon of the knockout mice. Intestinal claudin-7 knockout changed only the pFlux for small molecule solutes and did not completely destroy the TJ structure. Intestinal microorganisms entered the intestinal epithelial cells through the transcellular pathway, promoted intestinal inflammation, and then led to the occurrence of tumors. This model shows that loss of claudin-7 can increase the absorption of intestinal bacterial products and initiate colonic inflammation. Claudin-7 maintains the barrier function of the intestinal epithelium and regulates epithelial cell renewal.

Li et $\mathrm{al}^{41}$ successfully generated Cldn 7 intestinal conditional knockout mice with induction by tamoxifen to achieve temporally and spatially controllable induction. In $\mathrm{Cldn} 7^{-/-}$mice, atypical hyperplasia and adenoma formation were successfully induced by intraperitoneal injection of the carcinogen azoxymethane. Claudin-7 knockout mice have severe intestinal inflammation, epithelial cell detachment, necrosis, intestinal shortening, congestion, and edema. The intestinal epithelial structure was destroyed, necrotic glandular epithelial cells were found in the glandular cavity, and the basement membrane intercellular gap showed obvious loosening. H\&E staining showed connective tissue hyperplasia and breaks in the intestinal muscle for the formation of tumors. The research indicated that claudin-7 plays a significant role in maintaining intestinal homeostasis and tumorigenesis, and it may be a new tumor suppressor marker.

The three mouse models were tested for the effects of claudin-7 knockdown on intestinal inflammation and tumors. The claudin-7 general knockout mouse model showed a deadly phenotype with a short survival time and could not be further used to define the role of claudin-7 in CRC occurrence and development. With improvements in technology, the construction of a knockout mouse model of inducible conditional claudin-7 in the intestine will make further research possible. Claudin-7 knockout mice exhibit severe intestinal inflammation, increased paracellular permeability, a dysfunctional intestinal barrier, and successful induction of intestinal tumors, indicating that claudin-7 has protective effects on intestinal epithelial cells, maintains intestinal homeostasis, and may play a role in tumor inhibition.

\section{Claudin-7 and cancer: causal correlation}

As a critical protein in epithelial cells, abnormal expression and distribution of claudin-7 have been reported in a variety of human malignancies, including lung, colon, ovarian, breast, gastric, esophageal, and prostate cancers and has been associated with cancer progression and metastasis. ${ }^{11}$ As shown in Table 1, we summarized the expression profile of claudin-7 in different cancers. The dysregulation of claudin-7 expression and localization have the potential to 
Table I Dysregulated claudin-7 expression in diverse cancers

\begin{tabular}{|c|c|c|c|}
\hline Cancer type & Methodology & Expression change & Reference \\
\hline Lung cancer & IHC, DICFM & Downregulation & $12,40,45,46$ \\
\hline Esophageal cancer & $\mathrm{IHC}$ & Downregulation & $47-49$ \\
\hline Oral squamous cell carcinoma & $\mathrm{IHC}$ & Downregulation & 50 \\
\hline Breast cancer & $\mathrm{IHC}$ & Downregulation & 51,52 \\
\hline Endometrial cancer & $\mathrm{IHC}$ & Downregulation & 53 \\
\hline Prostate cancer & $\mathrm{IHC}$ & Downregulation & 54 \\
\hline Thyroid cancer & $\mathrm{IHC}$ & Downregulation & 55 \\
\hline Pancreatic cancer & $\mathrm{IHC}$ & Downregulation & 56 \\
\hline Colorectal cancer & IHC, ELISA, WB & Downregulation & $73-82$ \\
\hline Lung adenocarcinoma & $\mathrm{IHC}$ & Upregulation & 43 \\
\hline Esophageal adenocarcinoma & IHC, microarray analysis & Upregulation & 57 \\
\hline Nasopharyngeal carcinoma & IHC, WB & Upregulation & 58,59 \\
\hline Triple-negative breast cancer & $\mathrm{IHC}$ & Upregulation & 44,60 \\
\hline Gastric cancer & IHC, RT-qPCR & Upregulation & 61,62 \\
\hline Liver cancer & $\mathrm{IHC}$ & Upregulation & $17,64,65$ \\
\hline Thyroid carcinomas & RT-qPCR, IHC & Upregulation & 66,67 \\
\hline Prostate cancer & $\mathrm{IHC}$ & Upregulation & 68 \\
\hline Pancreatic cancer & WB, IHC & Upregulation & 69 \\
\hline Cervical adenocarcinoma & $\mathrm{IHC}$ & Upregulation & 70,71 \\
\hline Ovarian cancer & RT-qPCR, IHC, WB & Upregulation & 72 \\
\hline Colorectal cancer & IHC, WB, RT-qPCR & Upregulation & $83-85$ \\
\hline
\end{tabular}

Abbreviations: DICFM, digital image classification, fragmentation index, morphological analysis; ELISA, enzyme-linked immunosorbent assay; IHC, immunohistochemistry; RT-qPCR, reverse-transcription quantitative polymerase chain reaction; WB, Western blot.

alter homeostasis by impairing the integrity of the epithelial barrier, disrupting the basic regulatory mechanisms of cells, such as proliferation, differentiation, and apoptosis. Ultimately, this dysfunction contributes to cell transformation and tumorigenesis. ${ }^{42}$ Accordingly, loss of claudin-7 is associated with loss of cell adhesion and tumor progression. ${ }^{38}$ However, some studies have shown that increased expression of claudin-7 may promote tumor cell migration, invasion, and metastasis. ${ }^{43,44}$ These findings suggest that claudin- 7 may have carcinogenic or tumor suppressive effects.

Claudin-7 downregulation is correlated with the biological characteristics of tumor cells and the prognosis of some tumor patients. Lu et al used immunohistochemistry (IHC) to analyze the expression and location of claudin-7 in normal lung tissues and various types of lung cancer, and the results showed that claudin-7 is weak or absent in lung cancer. ${ }^{40}$ Claudin-7 knockdown cells have high phosphorylation of ERK1/2; however, cell proliferation, invasive ability, and ERK1/2 phosphorylation were significantly reduced with the restoration of claudin- $7 .{ }^{45}$ Claudin-7 inhibits the phosphorylation and nuclear localization of protein kinase $\mathrm{B}$ (PKB/Akt) and its association with 3-phosphoinositide-dependent protein kinase-1 (PDK1) under normal conditions. The deletion of claudin-7 eliminated the ability to inhibit the interaction between PDK1 and Akt and caused sustained phosphorylation of Akt, resulting in cell proliferation disorders of lung squamous cell carcinoma. ${ }^{12}$ A new digital image classification, fragmentation index, morphological analysis method for quantitative analysis of the expression pattern of claudin-7 in lung cancer also showed that claudin-7 can be used as a biomarker for identifying lung cancer. ${ }^{46}$ Claudin-7 expression was significantly decreased in paracancerous tissues and corresponding metastatic lymph nodes in esophageal cancer. The expression was associated with invasion depth, lymphatic invasion, and lymph node metastasis, suggesting that claudin-7 may be a predictor of lymph node metastasis. Claudin-7 hypermethylation occurred at the invasive front of esophageal squamous cell carcinoma and may lead to heterogeneous and reduced expression of claudin- $7 .{ }^{47} \mathrm{Clau}-$ din-7 knockout in esophageal SCC cells resulted in decreased expression of E-cadherin and accelerated cell growth and invasion. The abnormal localization of claudin-7 promotes cell transformation and regulates E-cadherin expression during tumor progression. ${ }^{48,49}$ Loss of claudin-7 expression in oral squamous cell carcinoma was associated with high pathological grade, TNM staging, vascular invasion, and regional lymph node involvement. ${ }^{50}$ Claudin-7 expression was also significantly associated with histological grade and distant metastasis in breast cancer ${ }^{51,52}$ and endometrial cancer, ${ }^{53}$ and it had prognostic significance. Claudin-7 may be associated with a tubular arrangement of tumor epithelial cells. Claudin-7 deletion leads to tumors with decreased 
tubular arrangement. Claudin-7 also regulates cell proliferation, resulting in higher histological grade tumors. Furthermore, claudin-7 was downregulated in prostate cancer, ${ }^{54}$ thyroid cancer, ${ }^{55}$ and pancreatic cancer. ${ }^{56}$ Its downregulation in tumors may be regulated through multiple mechanisms such as promoter methylation. Loss of TJ proteins is an event well known to reduce cell adhesion, and the deletion of intercellular adhesion molecules has been associated with decreased cell cohesion, and cytoskeleton remodeling, which is conducive to tumor cell detachment and distant metastasis. Paracellular permeability disorders provide the nutrients and growth factors required for tumor cell growth, allowing tumor cells to survive in the course of long-lasting invasion and metastasis. Claudin-7 plays an important role in inhibiting cancer invasion and proliferation, and it may also be a potential molecular target for therapy.

Upregulation of claudin-7 was reported to promote the malignant phenotype of cancer. Claudin-7 expression in invasive adenocarcinoma was significantly higher than that in normal epithelial tissue and precancerous lesions. The upregulation of claudin-7 may contribute to tumor cell adhesion and metastasis through non-TJ functions that involve interactions with the surrounding lung adenocarcinoma matrix. ${ }^{43}$ Microarray analysis showed that claudin-7 mRNA levels were increased in esophageal adenocarcinoma. Overexpression of claudin-7 may be an early event of carcinogenesis, which provides a target for the diagnosis and targeted treatment of esophageal adenocarcinoma. ${ }^{57}$ Claudin-7 expression was markedly increased in undifferentiated and poorly differentiated nasopharyngeal carcinoma. Palmitoylated claudin-7 may support tumor cell metastasis by interacting with MMPs and CD147. However, knockdown of claudin-7 inhibits tumor invasion and metastasis. ${ }^{58,59} \mathrm{IHC}$ revealed high claudin-7 expression in triple-negative breast cancer, which correlated with low c-kit expression and a higher tumor grade and has been shown to be an independent predictor of low recurrence-free survival. ${ }^{44,60}$ The expression of claudin-7 in gastric cancer was also significantly upregulated and persisted during tumor progression. The overall survival time of patients with high claudin-7 expression was decreased. In addition, the expression of claudin-7 in intestinal-type adenocarcinoma patients was significantly higher than that in patients with diffuse-type adenocarcinoma, and the high claudin-7 expression in intestinal adenocarcinoma cells may support the carcinogenic conversion of intestinal metaplasia to adenocarcinoma. Claudin-7 executes an oncogenic function, promoting cancer cell invasion, proliferation, and EMT. ${ }^{61-63}$ Compared with normal controls, hepatocellular carcinoma and cirrhosis tissues showed dramatically increased claudin-7 expression. Multivariate analysis showed that claudin-7 was an independent negative prognostic factor. ${ }^{17,64,65}$ The complexes of claudin-7, EpCAM, and CD44 variants may be involved in the invasive phenotype of undifferentiated thyroid carcinoma, ${ }^{66}$ but some experiments have shown that claudin-7 expression is not significantly different between benign and malignant thyroid cancers and thus is not a subtyping marker. ${ }^{67}$ Claudin-7 expression in prostate cancer, ${ }^{68}$ pancreatic cancer, ${ }^{69}$ cervical adenocarcinoma, ${ }^{70,71}$ and ovarian cancer $^{72}$ was similarly upregulated, and its high expression was significantly associated with poor prognosis. The mechanism underlying the difference in claudin-7 expression in different tissues is not yet clear, but may be related to the specific tissue and functional differences of claudin-7. Nonpalmitoylated claudin-7, as a TJ protein, is involved in the formation of intercellular TJs and inhibits tumor progression and metastasis through TJ function. However, palmitoylated claudin-7 inhibits the formation of TJs and promotes the degradation of the extracellular matrix through its non-TJ function or interaction with MMPs, thereby accelerating the movement of tumor cells and promoting tumor progression.

\section{The dual relationship between claudin-7 and colorectal cancer}

Studies in which the expression and function of claudin-7 in CRC have been analyzed are listed in Table 2. Claudin-7 expression in CRC also has a dual role. IHC showed significantly lower claudin-7 staining intensity in CRC tissue than adjacent non-tumor tissue, ${ }^{73-78}$ and abnormal cytoplasmic localization was observed. ${ }^{73}$ There was an important association between claudin-7 expression and lymphovascular invasion, neurotropic invasion, lymphocyte status, and tumor grade. $^{74-76,79}$ A decrease in claudin-7 mRNA levels is an early event in the development of CRC that may contribute to the damage of the epithelial barrier in adenomas. ${ }^{78}$ The immunoreactivity of claudin-7 gradually declines with the progression of CRC stage and claudin-7 indirectly regulates cell adhesion and promotes cancer progression. ${ }^{80}$ Serum claudin-7 expression in patients with CRC was significantly reduced, as shown by enzyme-linked immunosorbent assay (ELISA), and correlated with high $\mathrm{T}$ stage and high carcinoembryonic antigen levels. ${ }^{81}$ Using IHC and WB analyses, Bhat et al found that claudin-7 was downregulated in CRC, and the low claudin-7 expression promotes EMT and tumor progression. ${ }^{82}$ The above-mentioned evidence indicates that claudin-7 may play a potential tumor suppressor role in the occurrence and development of CRC, and it can be used as 
Table 2 Claudin-7 expression and function in colorectal cancer

\begin{tabular}{|c|c|c|c|}
\hline Reference & Decreased expression with colorectal cancer progression & Reference & $\begin{array}{l}\text { Increased expression with colorectal } \\
\text { cancer progression }\end{array}$ \\
\hline 73 & $\begin{array}{l}\text { Hypomethylation of claudin-7 and dystopic cytoplasmic location lead to } \\
\text { cancer progression }\end{array}$ & 83 & $\begin{array}{l}\text { Increased expression is inversely } \\
\text { correlated with disease-free survival and } \\
\text { higher degree of apoptosis resistance }\end{array}$ \\
\hline 74 & $\begin{array}{l}\text { Suppression of claudin-7 expression related to the depth of tumor } \\
\text { invasion, histological grade, lymphovascular invasion, positive regional } \\
\text { lymph nodes, perineural invasion, and lymphocytic response }\end{array}$ & 84 & $\begin{array}{l}\text { Claudin-7 overexpression promotes a loss } \\
\text { of tumor cell polarization and contributes } \\
\text { to proliferation and tumorigenesis }\end{array}$ \\
\hline 76 & $\begin{array}{l}\text { Decreased expression correlated with cancer progression, no } \\
\text { associations with clinicopathological parameter }\end{array}$ & 85 & $\begin{array}{l}\text { High expression of claudin- } 7 \text { is related to } \\
\text { migration and invasion potential }\end{array}$ \\
\hline 77 & $\begin{array}{l}\text { Hypermethylation at the claudin- } 7 \text { promoter leads to a reduction in } \\
\text { claudin-7 expression that is correlated with the incidence of vessel } \\
\text { infiltration and clinicopathologic stage }\end{array}$ & & \\
\hline 78 & $\begin{array}{l}\text { A decreased claudin-7 mRNA level is an early event in colorectal } \\
\text { carcinogenesis }\end{array}$ & & \\
\hline 79 & $\begin{array}{l}\text { The CLDN7 rs } 4562 \text { genotype is related to tumor differentiation and } \\
\text { lymph node involvement in colon carcinoma }\end{array}$ & & \\
\hline 80 & Low expression of claudin-7 is correlated with poor prognosis & & \\
\hline 81 & $\begin{array}{l}\text { Decreased claudin-7 expression is correlated with high T stage and high } \\
\text { CEA levels }\end{array}$ & & \\
\hline 82 & Low claudin-7 expression promotes EMT and tumor progression & & \\
\hline
\end{tabular}

Abbreviations: CEA, carcinoembryonic antigen; EMT, epithelial-mesenchymal transition.

a biomarker for predicting the development, proliferation, and prognosis of tumors.

However, claudin-7 also has a protumorigenic role. Kuhn et $\mathrm{al}^{83}$ and Darido et $\mathrm{al}^{84}$ found that claudin-7 is expressed in the entire colon crypt, but its staining intensity and properties are different from basal to surface epithelium. Claudin-7 expression is weak in normal colon crypts but is strongly overexpressed and shows a change in localization in the CRC samples. A complex is formed by four molecules (claudin-7, EpCAM, CO-029, and CD44v6) in the glycolipid-rich microdomain of the basolateral membrane, and it is upregulated in patients with CRC and liver metastases. The coexpression of these four molecules was positively correlated with clinical diversity, apoptosis resistance, and disease-free survival. It can inhibit cell apoptosis, increase cell invasiveness, and promote tumor progression. ${ }^{83}$ The $\beta$-catenin/Tcf activity and cell proliferation were enhanced after overexpression of claudin-7 using stable transfection, which promoted tumor cell polarization and tumor formation in xenograft mice. ${ }^{84}$ Knockdown of claudin-7 expression in the CRC cells HT29 and SW948 reduced cell proliferation, sphere formation, and migration and enhanced cell cycle progression, significantly reducing drug resistance and tumor formation. ${ }^{85}$ These studies demonstrate that claudin-7 overexpression may promote the initiation and progression of CRC tumors.

\section{Claudin-7, colorectal cancer metastasis, and EMT}

Invasion and metastasis are the key factors affecting the prognosis of colon cancer patients and are the major causes of cancer-related death in CRC patients. ${ }^{86}$ The main cause of CRC-induced death is metastasis to the peritoneum, lymph nodes, and liver. ${ }^{87,88}$ Increasing evidence suggests that claudins are not only a prognostic indicator of primary tumors but also functional effectors of metastasis. ${ }^{38}$ Invasion and metastasis of tumors may interact with the EMT process to obtain cancer stem cells. ${ }^{89,90}$ EMT is crucial for embryonic development, tissue remodeling, wound repair, and stem cell differentiation; it is a very significant pathophysiological process. ${ }^{91,92}$ However, abnormal activation may be driving the spread of cancer cells. ${ }^{93}$ EMT is closely related to invasion and metastasis of tumor cells and cancer cells escape from host immunity. Cancer cells conduct cell-cell signal transduction through EMT, resulting in decreased intercellular adhesion and polarity and increased migration and movement, which finally lead to cancer metastasis. ${ }^{11,94,95}$ There is strong evidence that tumor cell motility is associated with EMT, and effective suppression of EMT can inhibit CRC metastasis and improve the survival rate of CRC patients. ${ }^{96}$ Some investigations have demonstrated that EMT plays a critical role in CRC progression and invasion. ${ }^{97-99}$ 
There is little research on claudin-7 and EMT in CRC. Bhat et a ${ }^{82}$ found that low claudin- 7 expression induced EMT and enhanced tumor growth and invasiveness in nude mice through a mouse model of CRC. This research demonstrated that claudin-7 is a tumor suppressor involved in CRC EMT. Increased claudin-7 expression in low-claudin-7 SW620 colon cancer cells induced epithelial differentiation and reduced the ability of tumor formation, whereas knockdown of claudin-7 in the HT-29 or DLD-1 cell lines induced EMT and increased tumorigenicity, tumor growth, and invasiveness in nude mice. However, in a contrasting report, high claudin-7 expression was found to promote EMT. Claudin-7 recruits EpCAM to the glycolipid-enriched membrane fragment (GEM) and changes its conformation after cleavage by EpEx. Presenilin 2 binds to and releases the transcription factor EpIC. Palmitoylation of cld7 enhances the efficacy of EpIC and increases the expression of EMTrelated transcription factors and mesenchymal proteins, such as FGF, TGF $\beta$, and N-cadherin. ${ }^{85,100}$ HT29-cld $7{ }^{\mathrm{kd}}$ and SW948-cld7 ${ }^{\mathrm{kd}}$ cells affect the recruitment of EpCAM and display decreased intercellular connections, growth, and holoclone and sphere formation, resulting in the delayed tumor growth and decreased migration. Flow analysis and WB showed that N-cadherin, vimentin, and the transcription factor Snail were downregulated after claudin-7 knockdown, and E-cadherin was significantly upregulated. These findings indicated that low claudin-7 expression induced mesenchymal-epithelial transition (MET), and the cell migration and invasion potential was severely affected. Claudin-7 knockdown reduced drug resistance, promoted caspase activation, and decreased the activation of the anti-apoptotic PI3K/Akt pathway, indicating that claudin-7 may play a carcinogenic role. Decreasing the expression of claudin-7 in CRC may reduce invasion and metastasis and may be helpful for the diagnosis and treatment of CRC. ${ }^{85}$

Regardless of whether claudin-7 promotes EMT or conversely promotes MET, there is an interaction between claudin-7 and EMT in CRC, and it is crucial to confirm the exact mechanism.

\section{The regulation of claudin-7 expression in cancer}

The differential expression of claudin-7 in CRC is regulated by multiple mechanisms. The precise molecular mechanisms involved in the progression of cancer are still largely unknown, and recent studies have shown that transcription factors, cytokines, growth factors, post-translational modifications, and epigenetic mechanisms affect claudin-7 expression. ${ }^{11}$ Snail, a transcriptional repressor that promotes the progression of EMT and cancer, inhibits promoter activity by binding directly to the E-box of promoter regions and inhibiting the expression of claudin-7. ${ }^{101}$ Kohno et al ${ }^{102}$ found that claudin-7 expression is positively regulated by embryonic liver fodrin 3 (ELF3), and ELF3 belongs to the E26 transformation-specific sequence (ETS) transcription factor family. Luciferase reporter assays showed that the binding site of the ETS family is in the claudin-7 gene promoter region ( $\sim 150 \mathrm{bps})$. Claudin-7 mRNA expression can be downregulated by inhibiting ELF3 expression by siRNA, and claudin-7 mRNA expression can be induced by the introduction of ELF3 in a cell line lacking claudin-7 expression. Hepatocyte nuclear factor- $4 \alpha(\mathrm{HNF}-4 \alpha)$ is localized in the nucleus and is involved in the regulation of intestinal epithelial barrier function in mice. ${ }^{103}$ Studies have shown that HNF- $4 \alpha$ expression is related to claudin- 7 in differentiated intestinal epithelial cells. HNF-4 $\alpha$ regulates the endogenous claudin-7 promoter and directly upregulates claudin-7 protein expression. Claudin-7 expression was reduced in intestinal epithelial cells when HNF-4 $\alpha$ was knocked out. ${ }^{39}$

Epigenetic mechanisms include DNA methylation, histone modifications, or microRNAs, which are the major regulatory mechanisms regulating claudin protein and tumors. ${ }^{11}$ Promoter methylation induces gene transcription silencing by preventing transcription factor binding to target sites. ${ }^{104}$ Bhat et $\mathrm{a}^{82}$ and Nakayama et $\mathrm{al}^{77}$ found that claudin-7 was significantly downregulated in CRC tissues, and apparent hypermethylation of promoter $\mathrm{CpG}$ islands appeared in $\mathrm{CRC}$ cell lines with low claudin-7 expression. Claudin-7 mRNA and protein expression increased after applying the methylation inhibitor 5-aza-2'-deoxycytidine (5-Aza-dC); however, the claudin-7 expression was significantly decreased by DNA methyltransferases 3a (DNMT3a), which further suggested that hypermethylation inhibited claudin-7 expression in CRC. Hahn-Strömberg et $\mathrm{al}^{73}$ used bisulfite pyrosequencing to analyze DNA methylation of claudin-1, -4 , and -7 in tumors and the adjacent non-neoplastic mucosa and found that claudin-1 was significantly hypomethylated in the tumor tissues, while claudin-7 methylation levels showed no significant difference between tumors and paracancerous tissues. Claudin gene hypomethylation seems to be related to the abnormal compartmentalization of TJ proteins. The aforementioned studies showed that abnormal methylation of the claudin-7 gene reduced the expression of claudin-7 and plays an important role in the occurrence and development of tumors.

In CRC, claudin-7 phosphorylation enhances paracellular permeability of epithelial cells, increases the entry of 
growth factors and nutrients into tumor cells, and results in abnormal localization of claudin-7. Palmitoylation of claudin-7 can affect the localization of claudin-7; interfere with the formation of cell-cell adhesion in TJs; promote the recruitment of integrins and EpCAM to the GEM; interact with cytoskeletal connexin, MMP14, CD147, and TACE; participate in drug resistance; and activate the PI3K/Akt anti-apoptotic pathway, further promoting tumor invasion and metastasis. ${ }^{69,105}$ APC mutation induces claudin-1 expression in colonic epithelial cells, while claudin-1 inhibits the expression of claudin-7. The loss of claudin-7 in turn destroys the association between claudin-1, claudin- 7 , and $\beta$-catenin. ${ }^{106} \mathrm{In}$ addition, claudin- 7 knockout mice show that the interaction of EpCAM with claudin is important in the gut. ${ }^{31,33}$ EpCAM regulates the constituents and function of TJs by regulating the intracellular localization and degradation of claudin-7. ${ }^{107}$ EpCAM recruits claudin-7 into TJs, and EpCAM knockout mice show downregulated claudin-7 expression and disruption of TJs. ${ }^{108,109}$ Matriptase-mediated cleavage of EpCAM in intestinal epithelial cells resulted in decreased stability of claudin-7, dysregulated homeostasis of intestinal epithelial cells, and inhibition of EpCAM cleavage, leading to increased expression of claudin-7. ${ }^{110}$ In contrast, claudin-7 recruits EpCAM into GEM and promotes the expression of EMT-related transcription factors. ${ }^{85}$ The Wnt/Tcf-4 pathway negatively regulates the expression of claudin-7, Tcf- 4 can repress claudin-7 expression and maintain the low claudin-7 level in colonic crypts, and the transcription factor Sox -9 was identified as the basic conditioning mediator. However, this regulation is lost in CRC, which may be due to the decreased activity of Sox-9, and claudin-7 overexpression promotes tumor cell polarization and contributes to tumor development. ${ }^{84}$

Claudin-7 expression is involved in many processes, and it is an important target for inhibition of tumor proliferation and invasion, and to study the mechanism of regulatory factors.

\section{Conclusion}

Claudin-7 not only plays an important role in normal tissues but also has been fully confirmed to have a crucial role in cancer development and metastasis. Claudin-7 expression is reduced or increased in a tissue-specific manner and is related to TJ damage, the degree of malignancy, local recurrence, and distant metastasis. Defining the specific function of claudin-7 in the formation of tumors has become a major research focus in the field of cell biology. Although current research on claudin-7 is still ongoing, its functional characteristics, regulatory mechanisms, interactions with other claudin protein members, and specific mechanisms in promoting tumor invasion and metastasis remain unclear. Further research of claudin-7 may suggest new ideas for early diagnosis, treatment, and prognosis of tumors.

\section{Acknowledgments}

This work was supported by the National Natural Science Foundation of China $(81372585 ;$ 81772557) and the Beijing Health System High Level Training Plan of Health Technical Personnel (2014-3-048). Wenjing Li is now affiliated with Binzhou Medical University Hospital, Binzhou, Shandong, People's Republic of China.

\section{Disclosure}

The authors report no conflicts of interest in this work.

\section{References}

1. Mármol I, Sánchez-de-Diego C, Pradilla Dieste A, Cerrada E, Rodriguez Yoldi MJ. Colorectal carcinoma: a general overview and future perspectives in colorectal cancer. Int J Mol Sci. 2017;18(1):197.

2. Siegel RL, Miller KD, Jemal A. Cancer statistics, 2018. CA Cancer J Clin. 2018;68(1):7-30.

3. Chen W, Sun K, Zheng R, et al. Cancer incidence and mortality in China, 2014. Chin J Cancer Res. 2018;30(1):1-12.

4. Marley AR, Nan H. Epidemiology of colorectal cancer. Int $J$ Mol Epidemiol Genet. 2016;7(3):105-114.

5. Singh AB, Sharma A, Dhawan P. Claudin family of proteins and cancer: an overview. J Oncol. 2010;2010(3):541957-11.

6. Hou J, Paul DL, Goodenough DA. Paracellin-1 and the modulation of ion selectivity of tight junctions. J Cell Sci. 2005;118(21):5109-5118.

7. van Itallie CM, Anderson JM. Claudins and epithelial paracellular transport. Annu Rev Physiol. 2006;68(1):403-429.

8. Ding L, Lu Z, Lu Q, Chen YH. The claudin family of proteins in human maligancy: a clinical perspective. Cancer Manag Res. 2013;5:367-375.

9. Singh A, Mishra AK, Ylaya K, Hewitt SM, Sharma KC, Saxena S. Wilms tumor-1, claudin-1 and ezrin are useful immunohistochemical markers that help to distinguish schwannoma from fibroblastic meningioma. Pathol Oncol Res. 2012;18(2):383-389.

10. Farkas AE, Capaldo CT, Nusrat A. Regulation of epithelial proliferation by tight junction proteins. Ann NY Acad Sci. 2012;1258(1):115-124.

11. Singh AB, Dhawan P. Claudins and cancer: fall of the soldiers entrusted to protect the gate and keep the barrier intact. Semin Cell Dev Biol. 2015;42:58-65.

12. Akizuki R, Shimobaba S, Matsunaga T, Endo S, Ikari A. Claudin-5, -7, and -18 suppress proliferation mediated by inhibition of phosphorylation of Akt in human lung squamous cell carcinoma. Biochim Biophys Acta. 2017;1864(2):293-302.

13. Zihni C, Mills C, Matter K, Balda MS. Tight junctions: from simple barriers to multifunctional molecular gates. Nat Rev Mol Cell Biol. 2016;17(9):564-580.

14. Krause G, Winkler L, Mueller SL, Haseloff RF, Piontek J, Blasig IE. Structure and function of claudins. Biochim Biophys Acta. 2008;1778(3):631-645.

15. Matter K, Balda MS. SnapShot: epithelial tight junctions. Cell. 2014;157(4):992-992.e1.

16. Leech AO, Cruz RG, Hill AD, Hopkins AM. Paradigms lost - an emerging role for over-expression of tight junction adhesion proteins in cancer pathogenesis. Ann Transl Med. 2015;3(13):184. 
17. Tsujiwaki M, Murata M, Takasawa A, et al. Aberrant expression of claudin-4 and -7 in hepatocytes in the cirrhotic human liver. Med Mol Morphol. 2015;48(1):33-43.

18. Tamura A, Tsukita S. Paracellular barrier and channel functions of TJ claudins in organizing biological systems: advances in the field of barriology revealed in knockout mice. Semin Cell Dev Biol. 2014;36:177-185

19. Osanai M, Takasawa A, Murata M, Sawada N. Claudins in cancer: bench to bedside. Pflugers Arch. 2017;469(1):55-67.

20. Günzel D, Yu ASL, As Y. Claudins and the modulation of tight junction permeability. Physiol Rev. 2013;93(2):525-569.

21. Angelow S, Ahlstrom R, Yu AS. Biology of claudins. Am J Physiol Renal Physiol. 2008;295(4):F867-F876.

22. Piontek J, Winkler L, Wolburg H, et al. Formation of tight junction: determinants of homophilic interaction between classic claudins. Faseb J. 2008;22(1):146-158.

23. van Itallie CM, Gambling TM, Carson JL, Anderson JM. Palmitoylation of claudins is required for efficient tight-junction localization. $J$ Cell Sci. 2005;118(7):1427-1436.

24. van Itallie CM, Tietgens AJ, Logrande K, Aponte A, Gucek M, Anderson JM. Phosphorylation of claudin-2 on serine 208 promotes membrane retention and reduces trafficking to lysosomes. J Cell Sci. 2012;125(20):4902-4912.

25. Garcia-Hernandez V, Quiros M, Nusrat A. Intestinal epithelial claudins: expression and regulation in homeostasis and inflammation. Ann NY Acad Sci. 2017;1397(1):66-79.

26. Pope JL, Bhat AA, Sharma A, et al. Claudin-1 regulates intestinal epithelial homeostasis through the modulation of Notch-signalling. Gut. 2014;63(4):622-634.

27. Ivanov AI, Nusrat A, Parkos CA. The epithelium in inflammatory bowel disease: potential role of endocytosis of junctional proteins in barrier disruption. Novartis Found Symp. 2004;263:115-124.

28. Muto S, Hata M, Taniguchi J, et al. Claudin-2-deficient mice are defective in the leaky and cation-selective paracellular permeability properties of renal proximal tubules. Proc Natl Acad Sci USA. 2010;107(17):8011-8016.

29. Tamura A, Kitano Y, Hata M, et al. Megaintestine in claudin-15-deficient mice. Gastroenterology. 2008;134(2):523-534.

30. Alexandre MD, Lu Q, Chen YH. Overexpression of claudin-7 decreases the paracellular $\mathrm{Cl}$ - conductance and increases the paracellular Na+ conductance in LLC-PK1 cells. J Cell Sci. 2005;118(12): 2683-2693.

31. Ding L, Lu Z, Foreman O, et al. Inflammation and disruption of the mucosal architecture in claudin-7-deficient mice. Gastroenterology. 2012;142(2):305-315.

32. Tatum R, Zhang Y, Salleng K, et al. Renal salt wasting and chronic dehydration in claudin-7-deficient mice. Am J Physiol Renal Physiol. 2010;298(1):F24-F34.

33. Tanaka H, Takechi M, Kiyonari H, Shioi G, Tamura A, Tsukita S. Intestinal deletion of Claudin-7 enhances paracellular organic solute flux and initiates colonic inflammation in mice. Gut. 2015;64(10): 1529-1538.

34. Oshima T, Miwa H, Joh T. Changes in the expression of claudins in active ulcerative colitis. J Gastroenterol Hepatol. 2008;23(Suppl 2):S146-S150.

35. Hagen SJ. Non-canonical functions of claudin proteins: beyond the regulation of cell-cell adhesions. Tissue Barriers. 2017;5(2):e1327839.

36. Gonzalez-Mariscal L, Namorado MC, Martin D, Sierra G, Reyes JL. The tight junction proteins claudin-7 and -8 display a different subcellular localization at Henle's loops and collecting ducts of rabbit kidney. Nephrol Dial Transplant. 2006;21(9):2391-2398.

37. Fujita H, Chiba H, Yokozaki H, et al. Differential expression and subcellular localization of claudin-7, $-8,-12,-13$, and -15 along the mouse intestine. J Histochem Cytochem. 2006;54(8):933-944.

38. Tabariès S, Siegel PM. The role of claudins in cancer metastasis. Oncogene. 2017;36(9):1176-1190.
39. Farkas AE, Hilgarth RS, Capaldo CT, et al. HNF $4 \alpha$ regulates claudin-7 protein expression during intestinal epithelial differentiation. Am J Pathol. 2015;185(8):2206-2218.

40. Lu Z, Kim DH, Fan J, et al. A non-tight junction function of claudin-7 - interaction with integrin signaling in suppressing lung cancer cell proliferation and detachment. Mol Cancer. 2015;14(1):120-135.

41. Li WJ, Xu C, Wang K, et al. Severe intestinal inflammation in the small intestine of mice induced by controllable deletion of claudin-7. Dig Dis Sci. 2018;63(5):1200-1209.

42. Krug SM, Schulzke JD, Fromm M. Tight junction, selective permeability, and related diseases. Semin Cell Dev Biol. 2014;36:166-176.

43. Yamada G, Murata M, Takasawa A, et al. Increased expressions of claudin 4 and 7 in atypical adenomatous hyperplasia and adenocarcinoma of the lung. Med Mol Morphol. 2016;49(3):163-169.

44. Constantinou C, Papadopoulos S, Karyda E, et al. Expression and clinical significance of claudin-7, PDL-1, PTEN, c-Kit, c-Met, c-Myc, ALK, CK5/6, CK17, p53, EGFR, Ki67, p63 in triple-negative breast cancer - a single centre prospective observational study. In Vivo. 2018;32(2):303-311.

45. Lu Z, Ding L, Hong H, Hoggard J, Lu Q, Chen Y-H. Claudin-7 inhibits human lung cancer cell migration and invasion through ERK/MAPK signaling pathway. Exp Cell Res. 2011;317(13):1935-1946.

46. Lu Z, Liu Y, Xu J, et al. Immunohistochemical quantification of expression of a tight junction protein, claudin-7, in human lung cancer samples using digital image analysis method. Comput Methods Programs Biomed. 2018;155:179-187.

47. Usami Y, Chiba H, Nakayama F, et al. Reduced expression of claudin-7 correlates with invasion and metastasis in squamous cell carcinoma of the esophagus. Hum Pathol. 2006;37(5):569-577.

48. Lioni M, Brafford P, Andl C, et al. Dysregulation of claudin-7 leads to loss of E-cadherin expression and the increased invasion of esophageal squamous cell carcinoma cells. Am J Pathol. 2007;170(2):709-721.

49. Xm L, Wang H, Zhu LL, Zhao RZ, Hl J. Genes regulating epithelial polarity are critical suppressors of esophageal oncogenesis. J Cancer. 2015;6(8):694-700.

50. Phattarataratip E, Sappayatosok K. Expression of claudin-5, claudin-7 and occludin in oral squamous cell carcinoma and their clinicopathological significance. J Clin Exp Dent. 2016;8(3):e299-306.

51. Bernardi MA, Logullo AF, Pasini FS, et al. Prognostic significance of CD24 and claudin-7 immunoexpression in ductal invasive breast cancer. Oncol. Rep. 2012;27(1):28-38.

52. Flores AR, Rêma A, Carvalho F, Lopes G, Faustino A, Dias Pereira P. Clinicopathological significance of immunoexpression of claudin-1 and claudin-7 in feline mammary carcinomas. J Comp Pathol. 2014;151(4):339-346.

53. Li X, Li Y, Qiu H, Wang Y. Downregulation of claudin-7 potentiates cellular proliferation and invasion in endometrial cancer. Oncol Lett. 2013;6(1):101-105.

54. Sheehan GM, Kallakury BV, Sheehan CE, Fisher HA, Kaufman RP, Ross JS. Loss of claudins-1 and -7 and expression of claudins-3 and -4 correlate with prognostic variables in prostatic adenocarcinomas. Hum Pathol. 2007;38(4):564-569.

55. Tzelepi VN, Tsamandas AC, Vlotinou HD, Vagianos CE, Scopa CD. Tight junctions in thyroid carcinogenesis: diverse expression of claudin-1, claudin-4, claudin-7 and occludin in thyroid neoplasms. Mod Pathol. 2008;21(1):22-30.

56. Alikanoglu AS, Gunduz S, Demirpence O, et al. Expression pattern and prognostic significance of claudin 1, 4 and 7 in pancreatic cancer. Asian Pac J Cancer Prev. 2015;16(10):4387-4392.

57. Montgomery E, Mamelak AJ, Gibson M, et al. Overexpression of claudin proteins in esophageal adenocarcinoma and its precursor lesions. Appl Immunohistochem Mol Morphol. 2006;14(1):24-30.

58. Liu H, Jiang F, Jia X, et al. Cycling hypoxia affects cell invasion and proliferation through direct regulation of claudin 1 / claudin 7 expression, and indirect regulation of P18 through claudin 7. Oncotarget. 2017;8(6):10298-10311. 
59. Suren D, Yildirim M, Kaya V, et al. Expression patterns of claudins 1, 4, and 7 and their prognostic significance in nasopharyngeal carcinoma. J Buon. 2015;20(1):212-217.

60. Jääskeläinen A, Soini Y, Jukkola-Vuorinen A, Auvinen P, Haapasaari K-M, Karihtala P. High-level cytoplasmic claudin 3 expression is an independent predictor of poor survival in triple-negative breast cancer. BMC Cancer. 2018;18(1):223.

61. Jun K-H, Kim J-H, Jung J-H, Choi H-J, Chin H-M. Expression of claudin-7 and loss of claudin-18 correlate with poor prognosis in gastric cancer. Int J Surg. 2014;12(2):156-162.

62. Park JY, Park KH, Oh TY, et al. Up-regulated claudin 7 expression in intestinal-type gastric carcinoma. Oncol Rep. 2007;18(2):377-382.

63. Wu Z, Shi J, Song Y, et al. Claudin-7 (CLDN7) is overexpressed in gastric cancer and promotes gastric cancer cell proliferation, invasion and maintains mesenchymal state. Neoplasma. 2018;65(03):349-359.

64. Bouchagier KA, Assimakopoulos SF, Karavias DD, et al. Expression of claudins-1, -4, -5, -7 and occludin in hepatocellular carcinoma and their relation with classic clinicopathological features and patients' survival. In Vivo. 2014;28(3):315-326.

65. Á H, Gyöngyösi B, Lotz G, et al. Increased expression of claudin-1 and claudin-7 in liver cirrhosis and hepatocellular carcinoma. Pathol Oncol Res. 2014;20(3):493-502.

66. Okada T, Nakamura T, Watanabe T, et al. Coexpression of EpCAM, CD44 variant isoforms and claudin-7 in anaplastic thyroid carcinoma. PLoS One. 2014;9(4):e94487.

67. Süren D, Yildirim M, Sayiner A, et al. Expression of claudin 1, 4 and 7 in thyroid neoplasms. Oncol Lett. 2017;13(5):3722-3726.

68. Kwon MJ. Emerging roles of claudins in human cancer. Int J Mol Sci. 2013;14(9):18148-18180.

69. Thuma F, Heiler S, Schnölzer M, Zöller M. Palmitoylated claudin7 captured in glycolipid-enriched membrane microdomains promotes metastasis via associated transmembrane and cytosolic molecules. Oncotarget. 2016;7(21):30659-30677.

70. Akimoto T, Takasawa A, Murata M, et al. Analysis of the expression and localization of tight junction transmembrane proteins, claudin-1, $-4,-7$, occludin and JAM-A, in human cervical adenocarcinoma. Histol Histopathol. 2016;31(8):921-931.

71. Cunniffe C, Brankin B, Lambkin H, Ryan F. The role of claudin-1 and claudin-7 in cervical tumorigenesis. Anticancer Res. 2014;34(6): 2851-2857.

72. Dahiya N, Becker KG. Wood WH 3rd, Zhang Y, Morin PJ. Claudin-7 is frequently overexpressed in ovarian cancer and promotes invasion. PLoS One. 2011;6(7):e22119.

73. Hahn-Strömberg V, Askari S, Ahmad A, Befekadu R, Nilsson TK. Expression of claudin 1, claudin 4, and claudin 7 in colorectal cancer and its relation with CLDN DNA methylation patterns. Tumor Biology. 2017;39(4):1010428317697569.

74. Süren D, Yıldırım M, Kaya V, et al. Loss of tight junction proteins (Claudin 1, 4,and 7) correlates with aggressive behavior in colorectal carcinoma. Med Sci Monit. 2014;20:1255-1262.

75. Wang X, Tully O, Ngo B, Zitin M, Mullin JM. Epithelial tight junctional changes in colorectal cancer tissues. ScientificWorldJournal. 2011;11(4):826-841.

76. Tokuhara Y, Morinishi T, Matsunaga T, et al. Nuclear expression of claudin-3 in human colorectal adenocarcinoma cell lines and tissues. Oncol Lett. 2018;15(1):99-108.

77. Nakayama F, Semba S, Usami Y, Chiba H, Sawada N, Yokozaki H. Hypermethylation-modulated downregulation of claudin-7 expression promotes the progression of colorectal carcinoma. Pathobiology. 2008;75(3):177-185.

78. Bornholdt J, Friis S, Godiksen S, et al. The level of claudin-7 is reduced as an early event in colorectal carcinogenesis. BMC Cancer. 2011;11(1):65-74.

79. Hahn-Strömberg V, Askari S, Befekadu R, Matthiessen P, Karlsson $\mathrm{S}$, Nilsson TK. Polymorphisms in the CLDN1 and CLDN7 genes are related to differentiation and tumor stage in colon carcinoma. APMIS. 2014;122(7):636-642.
80. Ding L, Wang L, Sui L, et al. Claudin-7 indirectly regulates the integrin/ FAK signaling pathway in human colon cancer tissue. $J$ Hum Genet. 2016;61(8):711-720.

81. Karabulut M, Alis H, Bas K, et al. Clinical significance of serum claudin-1 and claudin-7 levels in patients with colorectal cancer. Mol Clin Oncol. 2015;3(6):1255-1267.

82. Bhat AA, Pope JL, Smith JJ, et al. Claudin-7 expression induces mesenchymal to epithelial transformation (MET) to inhibit colon tumorigenesis. Oncogene. 2015;34(35):4570-4580.

83. Kuhn S, Koch M, Nübel T, et al. A complex of EpCAM, claudin-7, CD44 variant isoforms, and tetraspanins promotes colorectal cancer progression. Mol Cancer Res. 2007;5(6):553-567.

84. Darido C, Buchert M, Pannequin J, et al. Defective claudin-7 regulation by Tcf- 4 and Sox- 9 disrupts the polarity and increases the tumorigenicity of colorectal cancer cells. Cancer Res. 2008;68(11):4258-4268.

85. Philip R, Heiler S, Mu W, Büchler MW, Zöller M, Thuma F. Claudin-7 promotes the epithelial - mesenchymal transition in human colorectal cancer. Oncotarget. 2015;6(4):2046-2063.

86. Massagué J, Obenauf AC. Metastatic colonization by circulating tumour cells. Nature. 2016;529(7586):298-306.

87. Zarour LR, Anand S, Billingsley KG, et al. Colorectal cancer liver metastasis: evolving paradigms and future directions. Cell Mol Gastroenterol Hepatol. 2017;3(2):163-173.

88. Franko J, Shi Q, Meyers JP, et al. Prognosis of patients with peritoneal metastatic colorectal cancer given systemic therapy: an analysis of individual patient data from prospective randomised trials from the Analysis and Research in Cancers of the Digestive System (ARCAD) database. Lancet Oncol. 2016;17(12):1709-1719.

89. Sato R, Semba T, Saya H, Arima Y. Concise review: stem cells and epithelial-mesenchymal transition in cancer: biological implications and therapeutic targets. Stem Cells. 2016;34(8):1997-2007.

90. Wang L, Wu Y, Lin L, et al. Metastasis-associated in colon cancer-1 upregulation predicts a poor prognosis of gastric cancer, and promotes tumor cell proliferation and invasion. Int J Cancer. 2013;133(6):1419-1430.

91. Chen T, You Y, Jiang H, Wang ZZ. Epithelial-mesenchymal transition (EMT): a biological process in the development, stem cell differentiation, and tumorigenesis. J Cell Physiol. 2017;232(12):3261-3272.

92. Lamouille S, Xu J, Derynck R. Molecular mechanisms of epithelialmesenchymal transition. Nat Rev Mol Cell Biol. 2014;15(3):178-196.

93. Wei SC, Yang J. Forcing through tumor metastasis: the interplay between tissue rigidity and epithelial-mesenchymal transition. Trends Cell Biol. 2016;26(2):111-120.

94. Lee JY, Kong G. Roles and epigenetic regulation of epithelial-mesenchymal transition and its transcription factors in cancer initiation and progression. Cell Mol Life Sci. 2016;73(24):4643-4660.

95. Tsai JH, Yang J. Epithelial-mesenchymal plasticity in carcinoma metastasis. Genes Dev. 2013;27(20):2192-2206.

96. Gonzalez DM, Medici D. Signaling mechanisms of the epithelialmesenchymal transition. Sci Signal. 2014;7(344):re8.

97. Cao H, Xu E, Liu H, Wan L, Lai M. Epithelial-mesenchymal transition in colorectal cancer metastasis: a system review. Pathol Res Pract. 2015;211(8):557-569.

98. Zhu QC, Gao RY, Wu W, Qin HL. Epithelial-mesenchymal transition and its role in the pathogenesis of colorectal cancer. Asian Pac J Cancer Prev. 2013;14(5):2689-2698.

99. Sipos F, Galamb O. Epithelial-to-mesenchymal and mesenchymal-toepithelial transitions in the colon. World J Gastroenterol. 2012;18(7): 601-608.

100. Maetzel D, Denzel S, Mack B, et al. Nuclear signalling by tumourassociated antigen EpCAM. Nat Cell Biol. 2009;11(2):162-171.

101. Feng J, Cen J, Li J, et al. Histone deacetylase inhibitor valproic acid (VPA) promotes the epithelial mesenchymal transition of colorectal cancer cells via up regulation of Snail. Cell Adh Migr. 2015;9(6):495-501.

102. Kohno Y, Okamoto T, Ishibe T, et al. Expression of claudin7 is tightly associated with epithelial structures in synovial sarcomas and regulated by an Ets family transcription factor, ELF3. J Biol Chem. 2006;281(50):38941-38950. 
103. Babeu JP, Boudreau F. Hepatocyte nuclear factor 4-alpha involvement in liver and intestinal inflammatory networks. World J Gastroenterol. 2014;20(1):22-30.

104. Esteller M, Corn PG, Baylin SB, Herman JG. A gene hypermethylation profile of human cancer. Cancer Res. 2001;61(8):3225-3229.

105. Heiler S, Mu W, Zöller M, Thuma F. The importance of claudin-7 palmitoylation on membrane subdomain localization and metastasispromoting activities. Cell Commun Signal. 2015;13:29.

106. Singh AB, Uppada SB, Dhawan P. Claudin proteins, outside-in signaling, and carcinogenesis. Pflugers Arch. 2017;469(1):69-75.

107. Wu CJ, Mannan P, Lu M, Udey MC. Epithelial cell adhesion molecule (EpCAM) regulates claudin dynamics and tight junctions. J Biol Chem. 2013;288(17):12253-12268.
108. Lei Z, Maeda T, Tamura A, et al. EpCAM contributes to formation of functional tight junction in the intestinal epithelium by recruiting claudin proteins. Dev Biol. 2012;371(2):136-145.

109. Mueller JL, Mcgeough MD, Peña CA, Sivagnanam M. Functional consequences of EpCam mutation in mice and men. Am J Physiol Gastrointest Liver Physiol. 2014;306(4):G278-G288.

110. Wu CJ, Feng X, Lu M, Morimura S, Udey MC. Matriptase-mediated cleavage of EpCAM destabilizes claudins and dysregulates intestinal epithelial homeostasis. J Clin Invest. 2017;127(2):623-634.

\section{Publish your work in this journal}

Cancer Management and Research is an international, peer-reviewed open access journal focusing on cancer research and the optimal use of preventative and integrated treatment interventions to achieve improved outcomes, enhanced survival and quality of life for the cancer patient. The manuscript management system is completely online and includes a very quick and fair peer-review system, which is all easy to use. Visit http://www.dovepress.com/testimonials.php to read real quotes from published authors. 